\title{
CHECK-LIST OF THE TASMANIAN LIVERWORTS
}

\author{
by D. A. Ratkowsky
}

RATKOWSKY, D.A., 1987 (30:vi): Check-list of the Tasmanian liverworts. Pap. Proc. R. Soc. Tasm., 121: 153-158. https://doi.org/10.26749/rstpp.121.153 ISSN 0080-4703. 117 York Street, Dynnyrne, Tasmania 7005

A check-list of the Tasmanian liverworts is presented in an at tempt to include all the species (282) that are presently believed to occur in Tasmania. The Lepidoziaceae is viewed as the family most in need of revision in southern Australia today.

Key Words: Tasmania, liverworts.

\section{INTRODUCTION}

It has been three score and ten years since the last major revision of the Tasmanian hepatic flora was published (Rodway 1917). Since that time, many advances have been made in our knowledge of the liverworts in this region. Nevertheless, there has been no systematic work to replace Rodway's study, although it is now widely recognised that many species in that work are synonyms, and that several other species have been discovered in the past seven decades which are absent from that work.

A valuable paper on the liverworts of the whole of Australia is the annotated list of binomials and check-list of published species by Scott \& Bradshaw (1985). That study was the starting point for the present paper, as it dealt critically with all the species names that were proposed up to about 1983.

\section{RESULTS AND DISCUSSION}

The check-list of Tasmanian liverworts is presented in table 1 . The arrangement of orders and families follows that of Scott \& Bradshaw (1985), although the spelling of two families, Balantiopsaceae and Chaetophyllopsaceae, have been altered from their spelling in that work on the advice of Dr R. Grolle (pers. comm.). Within families, genera are listed in alphabetical order, as are species within a genus.

A total of 282 species appears in the list in table 1 . This has to be considered to be a tentative list of the liverwort species of Tasmania, as several families, or genera within fa milies, require revision. For the Anthocerotales, a complete revision of this order of plants in Australia needs to be undertaken. For the order Metzgeriales, the genus Metzgeria is the most problematic, requiring revision. In the Aneuraceae, the genus Riccardia und oubtedly has some further undescribed species. Fossombronia is an extremely difficult genus to identify to species level and the Tasmanian list of species for this genus is likely to be incomplete or erroneous. In the Pallaviciniaceae, the check-list follows the recent opinion of $\mathrm{Dr} \mathrm{R}$. Grolle (pers. comm.), who currently believes, based on his herbarium studies, that in the genera Pallavicinia and/or Jensenia, only Pallavicinia rubristipa and $P$. xiphoides actually occur in Tasmania, with all records of Jensenia connivens (Col.) Grolle, J. pisicolor (Hook. f. \& Taylor) Grolle and Pallavicinia lyelli (Hook.) S.F. Gray from Tasmania being wrong. Symphyogyna rhodina has recently been rediscovered in Tasmania (collected by A.V. Ratkowsky), but study is needed to determine whether or not this species is another form of the highly polymorphic $S$. podophylla. Also, the type of $S$. interrupta needs examination.

In the order Marchantiales, a family that needs critical study is Ricciaceae. Although the genus Riccia in Australia was revised by NaThalang (1980), Tasmania was omitted from consideration. Thus, the species of Riccia in this checklist are preceded by a question mark and their identity must be viewed as doubtful. In Aytoniaceae, Dr R. Grolle (pers. comm.) asserts that Asterella australis (Tayl.) Verd., formerly believed to occur in Tasmania, is endemic to New Zealand.

The Jungermanniales is by far the largest order of liverworts and the one with the most pressing taxonomic problems. The situation with respect to Lepidoziaceae is serious, as there does not appear to be any hepaticologist currently working on a revision of this difficult family. The problem genera are Bazzania, Kurzia and Telaranea. Although Bazzania monilinervis is a distinctive species, $B$. adnexa and $B$. involut $a$ may be synonyms of a single very variable species. Although Kurzia and Telaranea appear in the list with nine species each, it is likely that some of the names in each 


\section{TABLE 1}

\section{Check-list of Tasmanian liverworts.}

\section{ANTHOCEROTALES}

Anthocerotaceae

Anthoceros laevis L.

Megaceros longispirus (Carringt. \& Pears.) Steph.

\section{METZGERIALES}

Aneuraceae

Aneura alterniloba (Hook. f. \& Tayl.) Hook. f. \& Tayl.

A. rodwayi Hewson

Riccardia aequicellularis (Steph.) Hewson

$R$. alcicornis (Hook. f. \& Tayl.) Trev.

$R$. bipinnatifida (Col.) Hewson

$R$. cochleata (Hook. f. \& Tayl.) Kuntze

$R$. colensoi (Steph.) Martin

$R$. crassa (Schwaegr.) Carringt. \& Pears.

$R$. eriocaula (Hook.) Besch. \& Massal.

$R$. gracilis (Steph.) Schust.

$R$. longiflora (Steph.) Hewson

$R$. minima Carringt. \& Pears.

$R$. nitida (Col.) Hodgs.

$R$. rupicola (Steph.) Hewson

$R$. wattsiana (Steph.) Hewson

Fossombroniaceae

Fossombronia intestinalis Tayl.

$F$. pusilla $($ L.) Nees

Hymenophytaceae

Hymenophyton flabellatum (Labill.) Dum. ex Trev.

H. leptopodum (Hook. f. \& Tayl.) Evans

Pallaviciniaceae

Pallavicinia rubristipa Schiffn.

P. xiphoides (Hook. f. \& Tayl.) Trev.

Podomitrium phyllanthus (Hook.) Mitt.

Symphyogyna interrupta Carringt. \& Pears.

S. podophylla (Thunb.) Mont. \& Nees

S. rhodina (Hook. f. \& Tayl.) Tayl.

\section{Metzgeriaceae}

Metzgeria atrichoneura Spruce

M. australis Steph.

$M$. colensoi Steph.

$M$. conjugata Lindb.

$M$. decipiens (Massal.) Schiffn. \& Gott.

$M$. densiseta Steph.

M. furcata (L.) Dum.

M. leptoneura Spruce
$M$. pinnata Steph

M. saccata Mitt.

\section{Treubiaceae}

Treubia lacunosa (Col.) Prosk.

T. tasmanica Schust. \& Scott

Vandiemeniaceae

Vandiemenia ratkowskiana Hewson

\section{MARCHANTIALES}

Aytoniaceae

Asterella drummondii (Hook. f. \& Tayl.) Schust. A. tasmanica (Steph.) Schust.

A. tenera (Mitt.) Schust.

Reboulia hemisphaerica (L.) Raddi

Marchantiaceae

Lunularia cruciata (L.) Dum.

Marchantia berteroana Lehm. \& Lindenb.

M. foliacea Mitt.

M. polymorpha L. var. aquatica Nees

Targioniaceae

Targonia hypophylla $\mathrm{L}$.

Ricciaceae

(?) Riccia crassa Steph.

(?) R. tasmanica Steph. ex Rodw.

(?)R. weymouthiana Steph. ex Rodw.

Ricciocarpos natans (L.) Corda

Monocleaceae

Monoclea forsteri Hook.

\section{CALOBRYALES}

Haplomitriaceae

Haplomitrium gibbsiae (Steph.) Schust.

\section{JUNGERMANNIALES}

Herbertaceae

Herbertus oldfieldianus (Steph.) Rodw.

Triandrophyllum subtrifidum (Hook. f. \& Tayl.) Fulf. \& Hatch.

Pseudolepicoleaceae

Isophyllaria attenuata (Rodw.) Hodgs.

Temnoma pulchellum (Hook.) Mitt.

T. townrowii Schust.

Trichotemnoma corrugatum (Steph.) Schust. 


\section{TABLE 1 (cont.)}

\section{Balantiopsaceae}

Acroscyphella phoenicorhiza (Grolle) Kitag. \& Grolle

Balantiopsis diplophylla (Hook. f. \& Tayl.) Mitt.

B. tumida Berggr.

Isotachis grandis Carringt. \& Pears.

I. intortifolia (Hook. f. \& Tayl.) Gott.

I. nigella Herz. [syn. Eoisotachis nigella (Herz.)

Schust.]

I. riparia Rodw.

\section{Ptilidiaceae}

Mastigophora flagellifera (Hook.) Steph.

Lepicoleaceae

Lepicolea scolopendra (Hook.) Dum. ex Trev.

Chaetophyllopsaceae

Chaetophyllopsis whiteleggei (Carringt. \& Pears.) Schust.

\section{Trichocoleaceae}

Eotrichocolea polyacantha (Hook. f. \& Tayl.)

Schust.

Trichocolea mollissima (Hook. f. \& Tayl.) Gott.

T. rigida Schust.

Lepidolaenaceae

Gackstroemia weindorferi (Herz.) Grolle

Lepidolaena brachyclada (Tayl. ex Lehm.) Trev.

L. clavigera (Hook.) Dum. ex Trev.

L. reticulata (Hook. f. \& Tayl.) Trev.

L. taylorii (Gott.) Trev.

Lepidoziaceae

Acromastigum anisostomum (Lehm. \& Lindenb.) Evans

A. cavifolium Schust.

A. colensoanum (Mitt.) Evans ex Reim.

A. mooreanum (Steph.) Hodgs.

Bazzania adnexa (Lehm. \& Lindenb.) Trev.

$B$. involuta (Mont.) Trev.

B. monilinervis (Lehm. \& Lindenb.) Trev.

B. novaezelandiae (Mitt.) Besch. \& Massa]

Hyalolepidozia longiscypha (Tayl.) Grolle

Hygrolembidium acrocladum (Berggr.) Schust.

$H$. australe (Steph.) Grolle

Isolembidium anomalum (Rodw.) Grolle

Kurzia cucullifolia (Steph.) Schust.

$K$. hippurioides (Hook. f. \& Tayl.) Grolle

$K$. quadriseta Grolle

K. saddlensis (Besch. \& Massal.) Grolle
$K$. setiformis (de Not,) Engel \& Schust.

$K$. sexflda (Steph.) urolle

$K$. temnomoides Schust.

$K$. tenax (Grev.) Grolle

$K$. verticillata (Carringt.) Grolle

Lepidozia concinna Col.

L. cupressina $(\mathrm{Sw}$.) Lindenb.

L. glaucophylla (Hook. f. \& Tayl.) Tayl.

L. laevifolia (Hook. f. \& Tayl.) Tayl.

L. microphylla (Hook.) Lindenb.

L. pendulina (Hook.) Lindenb.

L. procera Mitt.

L. ulothrix (Schwaegr.) Lindenb.

Neogrollea notabilis Hodgs.

Pseudocephalozia paludicola Schust.

Psiloclada clandestina Mitt.

Telaranea capilligera (Schwaegr.) Schust.

T. centipes (Tayl. ex Gott. et al.) Schust.

$T$. gottscheana (Lindenb.) Hodgs.

T. grossiseta (Steph.) Engel \& Schust.

T. herzogii (Hodgs.) Hodgs.

T. mooreana (Steph.) Schust.

T. patentissima (Hook. f. \& Tayl.) Hodgs.

T. praenitens (Lehm. \& Lindenb.) Hodgs.

T. tetradactyla (Hook. f. \& Tayl.) Hodgs.

Zoopsis argentea (Hook. f. \& Tayl.) Hook. f.

Z. leitgebiana (Carringt. \& Pears.) Bastow

Z. setulosa Leitg.

\section{Calypogeiaceae}

Calypogeia sphagnicola (H. Arnell \& Perss.) Warnst. \& Loeske

Jungermanniaceae

Anastrophyllum schismoides (Mont.) Steph.

Andrewsianthus perigonialis (Hook. f. \& Tayl.)

Schust.

Chandonanthus squarrosus (Hook.) Mitt.

Cryptochila grandiflora (Lindenb. \& Gott.)

Grolle

Cuspidatula monodon (Tayl.) Steph.

Jamesoniella colorata (Lehm.) Spruce ex Schiffn.

J. tasmanica (Hook. f. \& Tayl.) Steph.

Jungermannia inundata Hook, f. \& Tayl.

$J$. orbiculata (Col.) Grolle

Lophozia tasmanica Schust.

Gymnomitriaceae

Gymnomitrion incompletum (Gott.) Schust.

Herzogobryum aterrimum (Steph.) Grolle

H. erosum (Carringt. \& Pears.) Grolle

H. teres (Carringt. \& Pears.) Grolle 


\section{TABLE 1 (cont.)}

Scapaniaceae

Blephaxidophyllum vertebrale (Tayl.) Aongstr. B. xiphophyllum Grolle

Diplophyllum obtusifolium (Hook.) Dum.

\section{Schistochilaceae}

Pachyschistochila parvistipula (Rodw.) Schust. \& Engel

P. succulenta Engel \& Schust.

P. trispiralis (Schust.) Schust. \& Engel

Paraschistochila isotachyphylla Engel \& Schust.

$P$. pinnatifolia (Hook.) Schust.

P. tuloides (Hook. f. \& Tayl.) Schust.

Schistochila balfouriana (Hook. f. \& Tayl.) Steph.

S. lehmanniana (Lindenb.) Carringt. \& Pears.

S. pseudociliata Schust.

S. tasmanica Steph.

\section{Geocalycaceae}

Chiloscyphus amplectens (Mitt.) Engel \& Schust.

C. archeri Engel \& Schust.

C. australis Gott. et al.

C. biciliatus (Hook. f. \& Tayl.) Gott. et al.

C. bispinosus (Hook. f. \& Tayl.) Engel \& Schust.

C. echinellus (Lindenb. \& Gott.) Mitt.

C. excipulatus (Steph.) Engel \& Schust.

C. gippslandicus Engel \& Schust.

C. lentus (Hook. f. \& Tayl.) Engel \& Schust.

C. leucophyllus (Hook. f. \& Tayl.) Gott. et al.

C. muricatus (Lehm.) Engel \& Schust.

C. novae-zeelandiae (Lehm. \& Lindenb.) Engel \&

Schust.

C. okaritanus (Steph.) Engel \& Schust.

C. pallidius (Mitt.) Engel \& Schust.

C. planiusculus (Hook. f. \& Tayl.) Engel \& Schust.

C. semiteres (Lehm.) Lehm. \& Lindenb.

C. subporosus (Mitt.) Engel \& Schust.

C. variabilis (Steph.) Engel \& Schust.

C. villosus (Mitt. ex Steph.) Engel \& Schust.

Clasmatocolea marginata (Mitt. ex Steph.) Grolle

C. notophylla (Hook. f. \& Tayl.) Grolle

C. strongylophylla (Hook. f. \& Tayl.) Grolle

C. vermicularis (Lehm.) Grolle

C. verrucosa Engel

Geocalyx caledonicus Steph.

Hepatostolonophora paucistipula (Rodw.) Engel

$H$. rotata (Hook. f. \& Tayl.) Engel

Heteroscyphus allodontus (Hook. f. \& Tayl.) Engel

\& Schust.

$H$. argutus (Nees) Schiffn.

$H$. billardieri (Schwaegr.) Schiffn.

$H$. coalitus (Hook.) Schiffn.
$H$. colensoi (Mitt.) Schiffn.

H. conjugatus (Mitt.) Engel \& Schust.

H. cuneistipulus (Steph.) Schiffn.

H. cymbaliferus (Hook. f. \& Tayl.) Engel \& Schust.

$H$. decipiens (Gott.) Engel \& Schust.

H. fissistipus (Hook. f. \& Tayl.) Schiffn.

H. gunnianus (Mitt.) Engel \& Schust.

$H$. heterophyllus (Steph.) Engel \& Schust.

H. knightii (Steph.) Grolle

H. levieri (Steph.) Schiffn.

H. limosus (Carringt. \& Pears.) Schiffn.

H. lyallii (Mitt.) Schust.

H. multifidus (Steph.) Engel \& Schust.

H. physanthus (Hook. f. \& Tayl.) Schust.

$H$. simillimus (Steph.) Engel \& Schust.

H. sinuosus (Hook.) Schiffn.

H. supinus (Hook. f. \& Tayl.) Schust.

H. tasmanicus (Steph.) Engel \& Schust.

H. triacanthus (Hook. f. \& Tayl.) Schiffn

$H$. weymouthianus (Steph.) Schiffn.

Leptophyllopsis laxus (Mitt.) Schust.

Leptoscyphus expansus (Lehm.) Grolle

Saccogynidium decurvum (Mitt.) Grolle

Brevianthaceae

Brevianthus flavus (Grolle) Engel \& Schust.

Plagiochilaceae

Acrochila biserialis (Lehm. \& Lindenb.) Grolle Plagiochila annotina (Menz.) Lindenb.

$P$. baileyana Steph

$P$. circinalis (Lehm.) Lehm. \& Lindenb.

$P$. deltoidea Lindenb.

$P$. fasciculata Lindenb.

$P$. fruticella (Tayl.) Hook. f. \& Tayl.

$P$. fuscella (Tayl.) Tayl. \& Hook. f.

P. incurvicolla (Tayl.) Hook. f. \& Tayl.

P. lyallii Mitt.

P. pleurata (Tayl.) Hook. f. \& Tayl.

$P$. radiculosa Mitt.

P. ramosissima (Hook.) Lindenb.

$P$. ratkowskiana $\mathrm{H}$. Inoue

$P$. retrospectans Nees

P. stephensoniana Mitt.

P. strombifolia (Tayl.) Tayl. ex Lehm.

$P$. wattsii Steph. ex Rodw.

Plagiochilion conjugatus (Hook.) Schust.

Acrobolbaceae

Acrobolbus cinerascens (Lehm. \& Lindenb.)

Schiffn.

A. concinnus (Mitt.) Grolle 


\section{TABLE 1 (cont.)}

A. ochrophyllus (Hook. f. \& Tayl.) Schust. Goebelobryum unguiculatum (Hook. f. \& Tayl.) Grolle

Lethocolea squamata (Tayl.) Hodgs. Marsupidium setulosum Mitt.

M. surculosum (Nees) Schiffn. Tylimanthus pseudosaccatus Grolle T. tenellus (Hook. f. \& Tayl.) Mitt.

\section{Adelanthaceae}

Adelanthus bisetulus (Steph.) Grolle

A. falcatus (Hook.) Mitt.

A. occlusus (Hook. f. \& Tayl.) Carringt.

Cephaloziellaceae

Allisoniella nigra (Rodw.) Schust.

A. tasmanica Schust.

Cephaloziella arctica Bryhn \& Douin

C. exiliflora (Tayl.) Steph.

C. hirta (Steph.) Schust.

C. pulcherrima Schust.

\section{Radulaceae}

Radula aneurismalis (Hook. f. \& Tayl.) Nees

$R$, australiana Yamada

R. buccinifera (Hook. f. \& Tayl.) Tayl.

$R$. compacta Castle

$R$. multiamentula Hodgs.

$R$. novaehollandiae Hampe

R. plicata Mitt.

$R$. ratkowskiana Yamada

$R$. retroflexa Tayl.

$R$. tabularis Steph.

$R$. tasmanica Steph.

$R$. wattsiana Steph.

genus will be reduced to synonymy. A scholarly examination of this family, with 44 species in this check-list, is clearly the most urgent priority for the liverworts of southern Australia.

The species list for the Schistochilaceae stands on a firm basis, following the detailed recent study of Schuster \& Engel (1985). A firm basis for Geocalycaceae, the largest family of liverworts in Tasmania, will be provided when the study of this family to species level, being undertaken by Dr J. Engel and Prof. R.M. Schuster, is completed. At a generic level, Engel \& Schuster (1984) cogently argued that Lophocolea and Chiloscyphus could not be maintained as independent genera, so that species formerly known under Lophocolea are now given combinations in cnuoscyphus. in adaition,
Frullaniaceae

Frullania aterrima (Hook. f. \& Tayl.) Hook. f. \& Tayl.

F. clavata (Hook. f. \& Tayl.) Tayl.

$F$. engelii $\mathrm{Hatt}$

F. falciloba Tayl. ex Lehm.

F. monocera (Hook. f. \& Tayl.) Tayl.

F. pentapleura Tayl.

F. probosciphora Tayl.

F. rostrata (Hook. f. \& Tayl.) Hook. f. \& Tayl.

F. scandens Mont.

Lejeuneaceae

Acrolejeunea aulacophora (Mont.) Steph. Austrolejeunea olgae (Schust.) Schust.

Cheilolejeunea albovirens (Hook. f. \& Tayl.) Hodgs.

C. campbelliensis (Steph.) Schust.

C. comitans (Hook. f. \& Tayl.) Schust

C. mimosa (Hook. f. \& Tayl.) Schust.

Cololejeunea laevigata (Mitt.) Tilden

Colura pulcherrima Jov.-Ast.

C. saccophylla Hodgs. \& Herz.

Diplasiolejeunea plicatiloba (Hook. f. \& Tayl.) Grolle

Drepanolejeunea aucklandica Steph.

Harpalejeunea latitans (Hook. f. \& Tayl.) Grolle

Lejeunea cuspidistipula (Steph.) Steph.

L. drummondii Tayl.

L. norrisii Grolle

L. primordialis (Hook. f. \& Tayl.) Tayl.

Nephelolejeunea hamata Grolle

Siphonolejeunea nudipes (Hook. f. \& Tayl.) Herz.

their overview argued that many species formerly known under Chiloscyphus and all species known under Tetracymbaliella should now be transferred to Heteroscyphus. The names in table $\mathrm{l}$ reflect their viewpoint, although one must a wait their complete revision of all the species before one can be confident of which species do indeed occur in Tasmania. Of the remaining genera in Geocalycaceae in Tasmania, perhaps only Leptoscyphus needs clarification, as the name of the Tasmanian species, given here as $L$. expansus, must be viewed as tentative. The species of the genera Hepatostolonophora and Clasmatocolea are firmly based owing to recent detailed treatments (Engel 1979 and Engel 1980 respectively) 
The Plagiochilaceae in Tasmania is firmly based owing to the revision by Inoue \& Schuster (1971), as are Acrobolbaceae and Adelanthaceae, except for two questions that have plagued this writer: (i) Exactly how does Acrobolbus ochrophyllus differ from $A$. cinerascens, and (ii) Exactly how does Adelanthus occlusus differ from $A$. falcatus? In the Cephaloziellaceae, the genus Cephaloziella needs revision. So does Radula in the Radulaceae; the names presented in table 1 are an amalgamation of various sources, the most recent being Yamada (1984)

The Frullaniaceae is represented in Tasmania only by the genus Frullania. Knowledge of its species has been greatly aided by several recent papers of Hattori (1979a, 1979b, 1983) plus a personal communication from Dr $\mathrm{S}$. Hattori in which he (i) casts doubt on the occurrence of $F$. deplanata Mitt. in Tasmania,(ii) points out that $F$. engelii is closely related to, and possibly conspecific with, F. setchelii Pears. and F. pycnantha (Hook. f. \& Tayl.) Tayl., both supposed New Zealand endemics, (iii) asserts that $F$. gaudichaudii (Nees \& Mont.) Nees \& Mont. is a tropical species, probably not occurring in Australia and certainly not in Tasmania, (iv) believes that $F$. scandens may or may not be conspecific with $F$. mooreana Steph.; if not, the latter is the correct name for the Tasmanian species, and (v) does not recognise the occurrence of $F$. spinifera Tayl. in Tasmania.

The species list of the final family, the Lejeuneaceae, is very reliable due to the overview by Grolle (1982), which gave a firm basis for the list of species of that family.

\section{ACKNOWLEDGEMENTS}

The author is grateful for the taxonomic assistance given by Dr J.J. Engel (Chicago), Dr R. Grolle (Jena) and Dr S. Hattori (Nichinan). Without the helpful encouragement of Dr S.J. Jarman (Hobart), this paper would never have been written.

\section{REFERENCES}

ENGEL, J.J., 1979: Austral Hepaticae X. A revision of Hepatostolonophora Engel et Schust., nom. nov. (Hepaticae). J. Hattori Bot. Lab., 46: 91-108.

ENGEL, J.J., 1980: A monograph of Clasmatocolea (Hepaticae). Fieldiana, Botany, 3: 1-229.

ENGEL, J.J. \& SCHUSTER, R.M., 1984: An overview and evaluation of the genera of Geocalycaceae and subfamily Lophocoleoidae (Hepaticae). Nova Hedwigia, 39: 385-463.

GROLLE, R, 1982: Ubersicht der Lejeunaceae in Tasmanien. Wiss. Zeitschr. Friedrich-SchillerUniv. Jena, Math.-Nal. R., 31(2): 207-227.

HATTORI, S., 1979a, 1979b, 1983: A revision of the Australasian species of the genus Frullania. Hepaticae. J. Hattori Bot. Lab., I, 45: 323-363 II, 46: 119-153; III, 54: I33-182.

INOUE, H. \& SCHUSTER, R.M., 1971: A monograph of the New Zealand and Tasmanian Plagiochilaceae. J. Hattori Bot. Lab., 34: 1-225.

NA-THALANG, O., 1980: A revision of the genus Riccia (Hepaticae) in Australia. Brunonia, 3: 61-140.

RODWAY, L., 1917: Tasmanian Bryophyta. II. Hepatics. Pap. Proc. R. Soc. Tasm. (1916): 51-143. (Issued separately in 1916 as a single publication by the Royal Society of Tasmania: $93 \mathrm{pp}$.)

SCHUSTER, R.M. \& ENGEL, J.J., 1985: Austral hepaticae. V(2). Temperate and subantarctic Schistochilaceae of Australasia. J. Hattori Bot. Lab., 58: 255-539.

SCOTT, G.A.M. \& BRADSHAW, J.A., 1985: Australian liverworts (Hepaticae): annotated list of binomials and check-list of published species with bibliography. Brunonia, 8: 1-171.

YAMADA, K., 1984: Tasmanian species of Radula (Hepaticae) collected by Mrs. A.V. Ratkowsky. J. Jap. Bot., 59(3): 91-96.

(accepted 6 April 1987) 